\section{Tee-Immuntherapie}

ontaktallergien gegen Kreuzblütler wie Kamille, Ringelblume oder Arnika sind in Mitteleuropa relativ häufig, betroffen sind vor allem Freunde von Phytotherapie oder Naturkosmetik sowie Gärtner und Floristen. Verantwortlich für die Sensibilisierungen sind meist die Sesquiterpenlaktone dieser Pflanzen. Doch kann man möglicherweise durch eine orale Toleranzinduktion solchen Allergien vorbeugen. Das ist zumindest die Schlussfolgerung aus einem großen Ost-WestVergleich, bei dem 6.172 Patienten in Singapur und 7.557 Patienten in London einem Patchtest mit einem 0,1\%-igen Sesquiterpenlaktongemisch unterzogen wurden. Die Unterschiede waren signifikant: Während nur 0,34\% der Asiaten Reaktionen zeigten, waren es bei den Engländern $1,37 \%$, eine schwere Reaktion

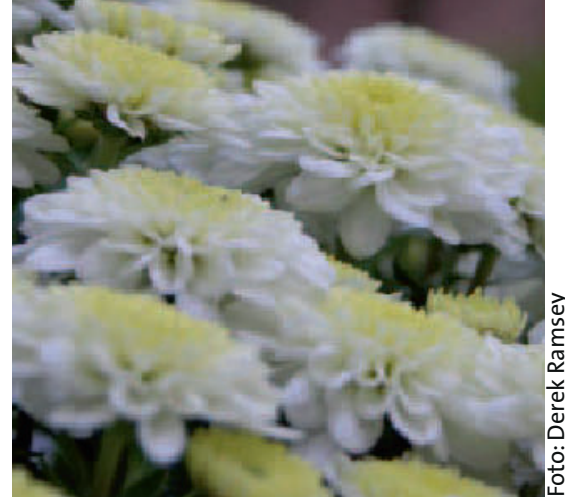

kam bei 0,06\% der Patienten aus Singapur und bei $0,89 \%$ der englischen Patienten vor. Das Immunsystem der Asiaten ist an die Sesquiterpenlaktone offensichtlich gewöhnt: Sie trinken von Kindesbeinen an regelmäßig Chrysanthemen-Tee. In Europa dienen die farbig blühenden Kompositen dagegen eher zur Verschönerung des Herbstgartens und als Ausgangsmaterial für Insektenvertilgungsmittel. $\quad b k$

Mak RK et al. Contact Dermatitis 2007; 57: $163-4$

\title{
Fiskus unerbittlich bei Zöliakie
}

E ine an Zöliakie erkrankte Frau hatte im Jahr 1996 insgesamt 3.192 DM für glutenfreie Nahrungsmittel ausgeben. Diese Aufwendungen wollte sie bei ihrer Einkommensteuererklärung als außergewöhnliche Belastung geltend machen. Das Finanzamt lehnte es jedoch ab, die Diätkosten steuerlich zu berücksichtigen. Dagegen klagte die Frau und verlor jetzt, neun Jahre später, auch in letzter Instanz beim Bundesfinanzhof. Nur unmittel- bare Krankheitskosten wie Arzneimittel seien als außergewöhnliche Belastung anerkannt, erklärten die Finanzrichter. Ausgaben für Nahrungsmittel zählten nicht dazu. Dieser Grundsatz gelte ohne Ausnahme - also sogar für Sonderdiäten, die, wie im konkreten Fall, weitgehend eine Behandlung mit Medikamenten ersetzten.

gri

Bundesfinanzhof, III R 48/04

\section{Mit Nanotechnologie gegen Allergien}

\begin{abstract}
E inen neuen Ansatz für eine antiallergische Therapie bietet die zurzeit boomende Nanotechnologie: Werden sphärische Kohlenstoffmoleküle, sogenannte Fullerene, in vitro mit Mastzellen und Blut-Basophilen inkubiert, unterbleibt nach Allergenstimulation die IgE-abhängige Mediatorfreisetzung nahezu komplett. Im Mausmodell der Mastzell-abhängigen Anaphylaxie stoppte die Vorabgabe von Fullerenen die Histamin-

$\mathrm{C}_{60}$-Moleküle diskutiert, freie Radikale zu neutralisieren. Sie übernehmen deren freie Elektronen und „verteilen" diese über ihre

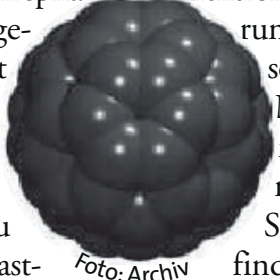
und 20 Benzolringe. Aufgrund der so erniedrigten Radikalzahl in den Mastzellen bleibt deren Aktivierung weitgehend aus. Hängt man nun noch bestimmte chemische Strukturen an die Nanokügelchen, finden sie spezifisch ihre Zielzellen im Organismus und können von diesen sogar phagozytiert werden.
\end{abstract} freisetzung nach Allergenprovokation. Als Wirkmechanismus wird die Fähigkeit der
Ryan JJ et al. J Immunol 2007; 179: 665-72

\section{Cave: Genvariante!}

ie Inzidenz des Plattenepithelkarzinoms steigt deutlich an. Sonnenlichtexpostition ist ein lange bekannter Risikofaktor, als weitere Auslöser wurden in jüngerer Zeit Immunsuppressiva wie Ciclosporin und Azathioprin identifiziert. Diskutiert wird derzeit auch ein Karzinom-induzierender Effekt von oral applizierten Glukokortikoiden. Ein besonders hohes Risiko haben dabei, so die Ergebnisse einer neuen populationsbasierten Fall-Kontroll-Studie, offenbar Träger einer relativ häufig vorkommenden Variante des GlukokortikoidRezeptor-Gens. Diese Genvariante, ein Bcl-Polymorphismus, trägt unter anderem zu einer erhöhten Glukokortikoidsensitivität bei. Die Datenlage ist aber insgesamt noch sehr unsicher, größere Studien müssen folgen.

$b k$

Patel AS et al. J Invest Dermatol 2007; 127: 1868-70

\section{Nase im Dauerstress}

D ie Nasen von Patienten mit allergischer Rhinitis bieten infektiösen Erregern einen guten Nährboden: die chronische Schleimhautentzündung, die vermehrte Expression des Adhäsionsmoleküls ICAM-1 und eine relative TH1-Schwäche machen die Patienten anfällig. Eine prospektive Studie mit einer sehr homogenen Studienkohorte bestätigt jetzt diesen Zusammenhang erneut: 624 männliche und weibliche italienische Marinesoldaten wurden über zwei Jahre hinsichtlich Häufigkeit und Schwere von Erkältungen untersucht. Tatsächlich waren die Seeleute mit allergischer Rhinitis signifikant häufiger und länger erkältet als ihre eher TH1-dominierten Kameraden. Gegen welche Erreger die Allergiepatienten besonders anfällig waren, wurde allerdings nicht untersucht.

$b k$

Cirillo I et al. Allergy 2007; 62: 1087-90 